\title{
Immunomodulatory and Anti-Inflammatory Phytochemicals for the Treatment of Inflammatory Bowel Disease (IBD)
}

\section{- Turning Strong Rationale into Strong Evidence? -}

\author{
Hamid Reza Sodagari ${ }^{1}$, Zahra Aryan ${ }^{2,3,4}$, Amir Hossein Abdolghaffari ${ }^{5,6,7,8 *}$, Nima \\ Rezaei ${ }^{4,9 *}$, Amirhossein Sahebkar ${ }^{10,11,12}$
}

${ }^{1}$ Young Researchers and Elite Club, Karaj Branch, Islamic Azad University, Karaj, Iran

'Students' Scientific Research Center, Tehran University of Medical Sciences, Tehran, Iran

${ }^{3}$ Pediatric Respiratory Diseases Education and Research Network (PRDERN), Universal Scientific Education and Research Network (USERN), Tehran, Iran

${ }^{4}$ Research Center for Immunodeficiencies, Children's Medical Center, Tehran University of Medical Sciences, Tehran, Iran

${ }^{5}$ Medicinal Plants Research Center, Institute of Medicinal Plants, ACECR, Tehran, Iran ${ }^{6}$ Students' Scientific Research Center, Tehran University of Medical Sciences, Tehran, Iran

${ }^{6}$ Department of Pharmacology, Pharmaceutical Sciences Branch, Islamic Azad University, Tehran, Iran

${ }^{7}$ Department of Toxicology and Pharmacology, Faculty of Pharmacy and Pharmaceutical Sciences Research Center, Tehran University of Medical Sciences, Tehran, Iran

${ }^{8}$ Gastrointestinal Pharmacology Interest Group (GPIG), Universal Scientific Education and Research Network (USERN), Tehran, Iran

${ }^{9}$ Network of Immunity in Infection, Malignancy and Autoimmunity (NIIMA), Universal Scientific Education and Research Network (USERN), Tehran, Iran

${ }^{10}$ Biotechnology Research Center, Pharmaceutical Technology Institute, Mashhad University of Medical Sciences, Mashhad, Iran

11 Neurogenic Inflammation Research Center, Mashhad University of Medical Sciences, Mashhad, Iran

${ }^{12}$ School of Pharmacy, Mashhad University of Medical Sciences, Mashhad, Iran

\section{Key Words}

inflammatory bowel disease, Crohn's disease, phytochemicals

Inflammatory bowel disease (IBD) comprises two types of chronic and relapsing intestinal inflammation conditions including Crohn's disease and ulcerative colitis [1]. Although the exact etiology of IBD remains elusive, the interaction of host's immune system with diet and microbiome of intestinal tract in genetically susceptible individuals seems to play a pivotal role in the pathogenesis of IBD [2]. Encoding regions for nucleotide oligomerization domain 2 (NOD2) and interleukin $23 \mathrm{~T}$ helper 17 (Th17) pathway are the most prominent genetic components of IBD pathogenesis [3,4]. NOD2 recognizes bacterial peptidoglycan and triggers the inflammatory cascade [5], and interleukin 23 is integral to immune defense against non-self-antigens and chronic intestinal inflammation [6]. On the other hand, break-

Received: Sep 21, 2017 Reviewed: Aug 09, 2018 Accepted: Nov 14, 2018

(c) This is an Open-Access article distributed under the terms of the Creative Common Attribution Non-Commercial License (http://creativecommons.org/licenses/by-nc/4.0/) which permits unrestricted noncommercial use, distribution, and reproduction in any medium, provided the original work is properly cited.

(2) This paper meets the requirements of KS X ISO 9706, ISO 9706-1994 and ANSI/NISO Z39.48-1992 (Permanence of Paper). down and alteration of normal microbiome increases the risk of intestinal colonization with pathogenic organisms and inflammatory diseases [7].

Dietary factors are known to influence gut microbiome and have the potential to shape the interplay between gut microbiome and immune responses involved in the pathogenesis of IBD [2]. Dietary factors can affect gut colonization of microorganisms in long term; they can mimic pathogenic antigens and trigger intracellular transduction and transcription pathways leading to modulation of inflammatory responses $[8,9]$. Exposure to stimuli such as reactive oxygen species, bacterial antigens and even innocent antigens activate nuclear factor (NF)-KB. This cascade results in the production of chemokines, pro-inflammatory cytokines, and infiltration of lymphocytes to the intestinal mucosa and disturbance of epithelial barrier leading to chronic intestinal inflammation. Phytochemicals including ellagic acid, curcumin, flavonoids, quercetin and green tea polyphenols can modulate NF-KB pathway [10-14]. Besides cytokine overproduction, overexpression of COX-2, the rate-limiting enzyme of 
prostaglandin production, is also involved in either acute or chronic intestinal inflammation. Phytochemicals such as grape juice and black raspberry powder have the ability to inhibit COX-2 and prostaglandin production $[15,16]$. Research on immunomodulatory and anti-inflammatory activities of phytochemicals in preventing and treating intestinal inflammation, and in modulating the gut microbiome and colitis symptoms is still at its infancy. Most of the evidence have come from animal studies [10-16], thus evidence from well-designed randomized controlled trials in this area are lacking. The shortcomings of available drugs to treat IBD and their side effects highlight a real need to additional therapies that could confer, either as alternative or adjunct, a better control of disease. In this context, phytochemicals are interesting candidates owing to their multimechanistic mode of action, potential safety, and wide availability $[1,2]$. Moreover, limited bioavailability of phytochemicals which is generally considered as an obstacle against their maximal systemic effects is less of a problem in IBD, as the site of action is intestine where the phytochemical is almost completely bioavailable upon oral use. While all these points emphasize the great therapeutic potential of phytochemicals for the treatment of IBD, important questions as to the dose-response association, clinical efficacy, precise mechanism(s) of action, and long-term tolerability still remain to be answered.

\section{Conflict of interest}

The authors declare that there are no conflicts of interest.

\section{References}

1. Hodson R. Inflammatory bowel disease. Nature. 2016;540(7634):S97-S97.

2. Ananthakrishnan AN. Nutritional Management of Inflammatory Bowel Diseases; 2016.

3. Barrett JC, Hansoul S, Nicolae DL, Cho JH, Duerr RH, Rioux JD, et al. Genome-wide association defines more than 30 distinct susceptibility loci for Crohn's disease. Nature Genetics. 2008;40(8):955-962.

4. Cho JH. The genetics and immunopathogenesis of inflammatory bowel disease. Nature Reviews Immunology. 2008;8(6):458-466.

5. Hedayat M, Netea MG, Rezaei N. Targeting of Tolllike receptors: a decade of progress in combating infectious diseases. The Lancet infectious diseases. 2011;11(9):702-712.

6. Abraham C, Cho J. Interleukin-23/Th17 pathways and inflammatory bowel disease. Inflammatory bowel diseases. 2009;15(7):1090-1100.

7. Kamada N, Seo SU, Chen GY, Nunez G. Role of the gut microbiota in immunity and inflammatory disease. Nat Rev Immunol. 2013;13(5):321-335.

8. González-Sarrías A, Espín JC, Tomás-Barberán FA, García-Conesa MT. Gene expression, cell cycle arrest and MAPK signalling regulation in Caco-2 cells exposed to ellagic acid and its metabolites, urolithins. Molecular nutrition \& food research. 2009;53(6):686-698.

9. Veiga P, Gallini CA, Beal C, Michaud M, Delaney ML,
DuBois A, et al. Bifidobacterium animalis subsp. lactis fermented milk product reduces inflammation by altering a niche for colitogenic microbes. Proceedings of the National Academy of Sciences. 2010;107(42):1813218137.

10. Yang F, Oz HS, Barve S, De Villiers WJ, McClain CJ, Varilek GW. The green tea polyphenol (-)-epigallocatechin-3-gallate blocks nuclear factor- $\mathrm{KB}$ activation by inhibiting IKB kinase activity in the intestinal epithelial cell line IEC-6. Molecular Pharmacology. 2001;60(3):528-533.

11. Rosillo MA, Sánchez-Hidalgo M, Cárdeno A, Aparicio-Soto M, Sánchez-Fidalgo S, Villegas I, et al. Dietary supplementation of an ellagic acid-enriched pomegranate extract attenuates chronic colonic inflammation in rats. Pharmacological Research. 2012;66(3):235242.

12. Holt PR, Katz S, Kirshoff R. Curcumin therapy in inflammatory bowel disease: a pilot study. Digestive diseases and sciences. 2005;50(11):2191-2193.

13. Vezza T, Rodríguez-Nogales A, Algieri F, Utrilla MP, Rodriguez-Cabezas ME, Galvez J. Flavonoids in inflammatory bowel disease: a review. Nutrients. 2016;8(4):211.

14. Dodda D, Chhajed R, Mishra J. Protective effect of quercetin against acetic acid induced inflammatory bowel disease (IBD) like symptoms in rats: Possible morphological and biochemical alterations. Pharmacological Reports. 2014;66(1):169-173.

15. Marchi P, Paiotti APR, Neto RA, Oshima CTF, Ribeiro DA. Concentrated grape juice $\left(\mathrm{G}_{8000}{ }^{\mathrm{mm}}\right)$ reduces immunoexpression of iNOS, TNF-alpha, COX-2 and DNA damage on 2, 4, 6-trinitrobenzene sulfonic acid-induced-colitis. Environmental toxicology and pharmacology. 2014;37(2):819-827.

16. Medda R, Lyros O, Schmidt JL, Jovanovic N, Nie L, Link BJ, et al. Anti inflammatory and anti angiogenic effect of black raspberry extract on human esophageal and intestinal microvascular endothelial cells. Microvascular research. 2015;97:167-180. 